\title{
Big Data Analytics for Business Intelligence in Accounting and Audit
}

\author{
Mui Kim Chu, Kevin Ow Yong \\ Singapore Institute of Technology, 10 Dover Drive, Singapore \\ Email: MuiKim.Chu@singaporetech.edu.sg, kevin.owyong@singaporetech.edu.sg
}

How to cite this paper: Chu, M. K., \& Yong, K. O. (2021). Big Data Analytics for Business Intelligence in Accounting and $\mathrm{Au}$ dit. Open Journal of Social Sciences, 9, 4252.

https://doi.org/10.4236/jss.2021.99004

Received: May 24, 2021

Accepted: August 29, 2021

Published: September 1, 2021

Copyright (อ 2021 by author(s) and Scientific Research Publishing Inc. This work is licensed under the Creative Commons Attribution International License (CC BY 4.0).

http://creativecommons.org/licenses/by/4.0/ (c) (i) Open Access

\begin{abstract}
Big data analytics represents a promising area for the accounting and audit professions. We examine how machine learning applications, data analytics and data visualization software are changing the way auditors and accountants work with their clients. We find that audit firms are keen to use machine learning software tools to read contracts, analyze journal entries, and assist in fraud detection. In data analytics, predictive analytical tools are utilized by both accountants and auditors to make projections and estimates, and to enhance business intelligence (BI). In addition, data visualization tools are able to complement predictive analytics to help users uncover trends in the business process. Overall, we anticipate that the technological advances in these various fields will accelerate in the coming years. Thus, it is imperative that accountants and auditors embrace these technological advancements and harness these tools to their advantage.
\end{abstract}

\section{Keywords}

Data Analytics, Machine Learning, Data Visualization, Audit Analytics

\section{Introduction}

Big data analytics has transformed the world that we live in. Due to technological advances, big data analytics enables new forms of business value and enterprise risk that will have an impact on the rules, standards and practices for the finance and accounting professions. The accounting and audit professionals are important players in harnessing the power of big data analytics, and they are poised to become even more vital to stakeholders in supporting data and insight-driven enterprises.

Data analytics can enable auditors to focus on exception reporting more efficiently by identifying outliers in risky areas of the audit process (IAASB, 2018). 
The advent of inexpensive computational power and storage, as well as the progressive computerization of organizational systems, is creating a new environment in which accountants and auditors must adapt to harness the power of big data analytics. In other applications, data analytics can help auditors to improve the risk assessment process, substantive procedures and tests of controls (Lim et al., 2020). These software tools have the potential to provide further evidence to assist with audit judgements and provide greater insights for audit clients.

In machine learning applications, the expectation is that the algorithm will learn from the data provided, in a manner that is similar to how a human being learns from data. A classic application of machine learning tools is pattern recognition. Facial recognition machine learning software has been developed such that a machine-learning algorithm can look at pictures of men and women and be able to identify those features that are male driven from those that are female driven. Initially, the algorithm might misclassify some male faces as female faces. It is thus important for the programmer to write an algorithm that can be trained using test data to look for specific patterns in male and female faces.

Because machine learning requires large data sets in order to train the learning algorithms, the availability of a vast quantity of high-quality data will expedite the process by allowing the programmer to refine the machine learning algorithms to be able to identify pictures that contain a male face as opposed to a female face. Gradually, the algorithm will be able to classify some general characteristics of a man (e.g., spotting a beard, certain differences in hair styles, broad faces) from those that belong to a woman (e.g., more feminine characteristics).

Similarly, it is envisaged that many routine accounting processes will be handled by machine learning algorithms or robotics automation processing (RPA) tools in the near future. For example, it is possible that machine learning algorithms can receive an invoice, match it to a purchase order, determine the expense account to charge and the amount to be paid, and place it in a pool of payments for a human employee to review the documents and release them for payment to the respective vendors.

Likewise, in auditing a client, a well designed machine learning algorithm could make it easier to detect potential fraudulent transactions in a company's financial statements by training the machine learning algorithm to successfully identify transactions that have characteristics associated with fraudulent activities from bona fide transactions. The evolution of machine learning is thus expected to have a dramatic impact on business, and it is expected that the accounting profession will need to adapt so as to better understand how to utilize such technologies in modifying their ways of working when auditing financial statements of their audit clients (Haq, Abatemarco, \& Hoops, 2020).

Predictive analytics is a subset of data analytics. Predictive analytics can be viewed as helping the accountant or auditor in understanding the future and provides foresight by identifying patterns in historical data. One of the most common applications of predictive analytics in the field of accounting is the compu- 
tation of a credit score to indicate the likelihood of timely future credit payments. This predictive analytics tool can be used to predict an accounts receivable balance at a certain date and to estimate a collection period for each customer.

Data visualization tools are becoming increasingly popular because of the way these tools help users obtain better insights, draw conclusions and handle large datasets (Skapoullis, 2018). For example, auditors have begun to use visualizations as a tool to look at multiple accounts over multiple years to detect misstatements. If an auditor is attempting to examine a company's accounts payable (AP) balances over the last ten years compared to the industry average, a data visualization tool like PowerBI or Tableau can quickly produce a graph that compares two measures against one dimension. The measures are the quantitative data, which are the company's AP balances versus the industry averages. The dimension is a qualitative categorical variable. The difference between data visualization tools from a simple Excel graph is that this information ("sheet') can be easily formatted and combined with other important information ("other sheets") to create a dashboard where numerous sheets are compiled to provide an overall view that shows the auditor a cohesive audit examination of misstatement risk or anomalies in the company's AP balances. As real-time data is streamed to update the dashboard, auditors could also examine the most current transactions that affect AP balances; thus, enabling auditor to perform continuous audit. With the realtime quality dashboard that provide real-time alerts, it enables collaboration among the audit team on a real-time continuous basis coupled with real-time supervisory review. Analytical procedures and test of transactions can be done more continually, and the auditor can investigate unusual fluctuations more promptly. The continuous review can also help to even out the workload of the audit team as the audit team members are kept abreast of the client's business environment and financial performance throughout the financial year.

The next section discusses machine learning applications to aid the audit process. Section 3 describes predictive analytics and how accountants and auditors use these tools to generate actionable insights for companies. Section 4 discusses data visualization and its role in the accounting and audit profession. Section 5 concludes.

\section{Machine Learning}

\subsection{Machine Learning in Audit Processes}

Machine learning is a subset of artificial intelligence that automates analytical model building. Machine learning uses these models to perform data analysis in order to understand patterns and make predictions. The machines are programmed to use an iterative approach to learn from the analyzed data, making the learning an automated and continuous process. As the machine is exposed to greater amount of data, more robust patterns are recognized. In turn, this iterative process helps to refine the data analysis process. Machine learning and traditional statistical analysis are similar in many aspects. However, while statistical analysis is based 
on probability theory and probability distributions, machine learning is designed to find that optimal combination of mathematical equations that best predict an outcome. Thus, machine learning is well suited for a broad range of problems that involve classification, linear regression, and cluster analysis.

The predictive reliability of machine learning applications is dependent on the quality of the historical data that has been fed to the machine. New and unforeseen events may create invalid results if they are left unidentified or inappropriately weighted. As a result, human biases can influence the use of machine learning. Such biases can affect which data sets are chosen for training the AI application, the methods chosen for the process, and the interpretation of the output. Finally, although machine learning technology has great potential, its models are still currently limited by many factors, including data storage and retrieval, processing power, algorithmic modeling assumptions, and human errors and judgment.

Machine learning technology for auditing is a very promising area (Dickey et al., 2019). Several of the Big 4 audit firms have machine learning systems under development, and smaller audit firms are beginning to benefit from improving viability of this technology. It is expected that auditing standards will adapt to take into account the use of machine learning in the audit process. Regulators and standard setters will also need to consider how they can incorporate the impact of this technology in their regulatory and decision making process. Likewise, educational programs will continue to evolve to this new paradigm. We foresee that more accounting programs with data analytics and machine learning specializations will become the norm rather than the exception.

Although there are certain limitations to the current capability of machine learning, it excels at performing repetitive tasks. Because an audit process requires a vast amount of data and has a significant number of task-related components, machine learning has the potential to increase both the speed and quality of audits. By harnessing machine-based performance of redundant tasks, it will free up more time for the auditors to undertake review and analytical work.

\subsection{Current Audit Use Cases}

Audit firms are already testing and exploring the power of machine learning in audits. One example is Deloitte's use of Argus, a machine learning tool that "learns" from every human interaction and leverages advanced machine learning techniques and natural language processing to automatically identify and extract key accounting information from any type of electronic document such as leases, derivatives contracts, and sales contracts. Argus is programmed with algorithms that allow it to identify key contract terms, as well as trends and outliers. It is highly possible for a well-designed machine to not just read a lease contract, identify key terms, determine whether it is a capital or operating lease, but also to interpret nonstandard leases with significant judgments (e.g., those with unusual asset retirement obligations). This would allow auditors to review and assess larger samples-even up to $100 \%$ of the documents, spend more time on judgemental areas 
and provide greater insights to audit clients, thus improving both the speed and quality of the audit process.

Another example of machine learning technology currently used by PricewaterhouseCoopers is Halo. Halo analyzes journal entries and can identify potentially problematic areas, such as entries with keywords of a questionable nature, entries from unauthorized sources, or an unusually high number of journal entry postings just under authorized limits. Similar to Argus, Halo allows auditors to test $100 \%$ of the journal entries and focusing only on the outliers with the highest risk, both the speed and quality of the testing procedures are significantly improved.

\subsection{Potential Machine Learning Applications}

Audit firms and academics are studying additional ways that machine learning can be used in financial statement audits, particularly in the risk assessment process. For example, machine learning technologies such as speech recognition could be used to examine and diagnose executive fraud interviews. The software can be used to identify situations when interviewees give questionable answers, such as "sort of" or "maybe," that suggest potential deceptive behavior. Significant delays in responses, which might also indicate deliberate concealment of information, can also be picked up by such speech recognition technology Facial recognition technologies can be applied toward fraud interviews as well. An AI software that uses facial recognition can help to identify facial patterns that suggest excess nervousness or deceit during entrant interviews. The assistance of speech and facial recognition technology in fraud interviews could certainly complement auditors and notify them when higher-risk responses warrant further investigation.

A study was done to assess risk based on machine learning by using a deep neural network (DNN) model to develop and test for a drive-off scenario involving an Oil \& Gas drilling rig. The results of the study show a reasonable level of accuracy for DNN predictions and a partial suitability to overcome risk assessment challenges. Perhaps such deep learning approach can be extended to auditing by training the model on past indicators of inherent risk, for the purpose of assessing risk of material misstatements. Data from various exogenous sources, such as forum posts, comments, conversations from social media, press release, news, management discussion notes, can be used to supplement traditional financial attributes to train the model to virtually assess the inherent risk levels (Paltrinieri et al., 2019).

The use of machine learning for risk assessment can also be applied to assessment of going concern risk. By studying the traits of companies that have gone under financial distress, a Probability of Default (PD) model can be developed, with the aim to quantify the going concern on a timelier basis. The predictive model requires an indicator of financial distress and a set of indicators that leverage on environmental and financial performance scanning to produce a PD that 
is dynamically updated according to firm performance (Martens et al., 2008).

The impact on businesses and the accounting profession will undoubtedly be significant in the near future. The major public accounting firms are focused on providing their customers with the expertise needed to deploy machine learning algorithms in businesses to accelerate and improve business decisions while lowering costs. In May 2018, PricewaterhouseCoopers announced a joint venture with eBravia, a contract analytics software company, to develop machine learning algorithms for contract analysis. Those algorithms could be used to review documents related to lease accounting and revenue recognition standards as well as other business activities, such as mergers and acquisitions, financings, and divestitures. In the area of advisory services, Deloitte has advised retailers on how they can enhance customer experience by using machine learning to target product and services based on past buying patterns. While the major public accounting firms may have the financial resources to invest in machine learning, small public accounting firms can leverage on these technological solutions and use prebuilt machine learning algorithms to develop expertise through their own implementations at a smaller scale.

\section{Data Analytics}

\subsection{Predictive Analysis in Accounting Processes}

Traditionally, accounting has focused more on fact-based, historical reporting. While this perspective helps executives in analyzing historical results so they can adjust their strategic and operational plans going forward, it does not necessarily help them better predict and more aggressively plan for the future.

Finding the right solution to enable a detailed analysis of financial data is critical in the transition from looking at the historical financial data to find predictors that enable forward-looking business intelligence (BI). A BI solution leverages on patterns in your data. Looking at consolidated data in an aggregate manner rather than in a piecemeal ad-hoc process from separate information systems provides an opportunity to uncover hidden trends and is a useful functionality for predictive analytics. For example, in customer relationship management (CRM) systems, improved forecasting is important in better planning for capacity peaks and troughs that directly impact the customer experience, response time, and transaction volumes.

Many accountants are already using data analytics in their daily work. They compute sums, averages, and percent changes to report sales results, customer credit risk, cost per customer, and availability of inventory. Accountants also are generally familiar with diagnostic analytics because they perform variance analyses and use analytic dashboards to explain historical results.

The various attempts to try to predict financial performance and leveraging on nonfinancial performance measures that might be good predictors of financial performance is expected to gain much traction in the coming years. This presents a great opportunity for accountants to provide a much more valuable role to 
management. Hence, accountants should further harness the power of data analytics to effectively perform their roles.

Predictive analytics and prescriptive analytics are important because they provide actionable insights for companies. Accountants need to increase their competence in these areas to provide value to their organizations. Predictive analytics integrates data from various sources (such as enterprise resource planning, point-of-sale, and customer relationship management systems) to predict future outcomes based on statistical relationships found in historical data using regression-based modeling. One of the most common applications of predictive analytics is the computation of a credit score to indicate the likelihood of timely future credit payments. Prescriptive analytics utilizes a combination of sophisticated optimization techniques (self-optimizing algorithms) to make recommendations on the most favorable courses of action to be taken.

The analytics skills that an accountant needs will differ depending on whether the accounting professional will produce or consume information. Analytics production includes sourcing relevant data and performing analyses, which is more suitable for junior-level accountants. Analytics consumption is using the insights gained from analytics in decision-making and is more relevant for senior-level roles. It is not expected that accountants need to retool to become data scientists or computer engineers to harness analytics tools. Nevertheless, it is most important that the audit and accounting professions become more proficient consumers of analytics to both enhance their current audit practice with available technologies as well as to support their client base in undertaking data analytics activities (Tschakert et al., 2016).

\subsection{Data Analytics Applications in Audit Processes}

Audit Data Analytics (ADAs) help auditors discover and analyze patterns, identify anomalies and extract other useful information from audit data through analysis, modeling and visualization. Auditors can use ADAs to perform a variety of procedures to gather audit evidence, to help with the extraction of data and facilitate the use of audit data analytics, and a tool to help illustrate where audit data analytics can be used in a typical audit program (McQuilken, 2019).

Governance, risk and control, and compliance monitoring systems commonly used by larger companies include systems developed by Oracle, SAP and RSA Archer. Oracle and SAP have application-side business intelligence systems centred on business warehouses. Lavastorm, Alteryx and Microsoft's SQL server provide advanced tools for specialists such as business analysts and, increasingly, for non-specialists. All these platforms are currently the preserve of large systems integrators, larger and mid-tier firm consultancies and specialist data analysts. It seems likely though, that over time these systems will move in-house or be provided as managed services. It also seems likely that companies such as CaseWare and Validis that currently provide data analytics services to larger and mid-tier firms, enabling those firms to offer data analytics services to their own clients.

Some businesses already analyze their own data in a similar manner to audi- 
tors. As these business analyses become deeper, wider, and more sophisticated, with a focus on risk and performance, it seems likely that they will align at least in part with the risks assessed by external auditors.

Data analytics is rooted in software originally developed in the early 2000s for data mining in the banking and retail sectors, and for design and modelling in financial services and engineering. What is phenomenal about this process is the volumes of data that can be handled efficiently on an industrial scale, and the speed of calculations being performed in a fraction of a second. The type of tasks such software can perform, and the connections it can make, dwarf what was previously possible. These technological improvements have facilitated the advances that we have seen in data analytics software (Davenport, 2016).

\subsection{Current Audit Use Cases}

By using data analytics procedures, accountants and auditors can produce highquality, statistical forecasts that help them understand and identify risks relating to the frequency and value of accounting transactions. Some of these procedures are simple, others involve complex models. Auditors using these models will exercise professional judgement to determine mathematical and statistical patterns, helping them identify exceptions for extended testing (Zabeti, 2019).

Auditors commonly use data analytics procedures to examine:

- Receivables and payables ageing;

- Analysis of gross margins and sales, highlighting items with negative margins;

- Analysis of capital expenditure versus repairs and maintenance;

- Matching of orders and purchases;

- Testing of journal entries.

Although data analytics techniques may not entirely substitute the traditional audit procedures and techniques, they can be powerful enablers which allow auditors to perform procedures and analysis which were not traditionally possible.

For example, a three-way match process is one of the most basic procedures in audit. Traditionally, auditors perform this procedure by way of sample testing as it is typically not realistic nor expected for auditors to vouch all transaction documents. Data analytics techniques now provide auditors the ability to analyze all the transactions which have been recorded. Hence, auditors can potentially filter and identify a specific class of transactions with unmatched items. Data analytics tools can also allow auditors to trace revenue transactions to debtors and the subsequent cash received and also analyze payments made after period end. This technique can relate the subsequent payments with the delivery dates extracted from the underlying delivery documents to ascertain if the payments relate to goods delivered before the period end or after the period end and also determine the amount of unrecorded liability.

\section{Data Visualization}

\section{Data Visualization in Accounting and Audit Processes}

The auditing and accounting professions have allocated a large amount of re- 
sources in understanding the impact of different data visualizations techniques in decision making and analytical procedures. As the technology evolves, and the size and volume of data is continuously growing, new ways to present information are emerging, it is vital for accounting and auditing research to examine newer data visualization techniques (Alawadhi, 2015).

The main objective of data visualization is to help users obtain better insights, draw better conclusions and eventually create hypotheses. This is achieved by integrating the user's perceptual abilities to the data analysis process, and applying their flexibility, creativity, and general knowledge to the large data sets available in today's systems. Data visualization involves several main advantages. It presents data in a concise manner. It also allows for faster data exploration in large data sets. Finally, data visualization tools are intuitive and do not require an understanding of complex mathematical or statistical algorithms.

New software is constantly being developed to help users work with the ever-increasing volume of data produced by businesses. More and more accounting firms and private businesses are using new BI tools such as Tableau, Power BI and QlikSense (Eaton \& Baader, 2018). Auditors have begun to use visualizations as a tool to look at multiple accounts over multiple years to detect misstatements. These tools can be used in risk analysis, transaction and controls testing, analytical procedures, in support of judgements and to provide insights. Many data analytics routines can now easily be performed by auditors with little or no management involvement. The ability to perform these analyses independently is important. Many routines can be performed at a very detailed level. The higher-level routines can be used for risk analysis to find a problem, while the more detailed analysis can be used to provide audit evidence and/or insights.

Another promising feature of data visualization tools relates to an audit engagement communication. With these tools, information can be summarized and presented in a way that is attentional and sufficient. A reader of the report will get the required information with a simple glance of a visual presentation. It is possible that an opinion will be much more powerful if is accompanied with a visualization of facts rather than statements describing the factors to support the opinion. Introducing visualization techniques can make reports easier to read and understand while focusing on the main figures of what an auditor is trying to report. While analyzing data is the crux of an external audit, it is critical that auditors know how to work with data. Doing so ensures they will better understand their client and plan a quality audit.

As the pace of innovation continues to increase, data visualization may become a necessary part of the job for many accountants and auditors. Accountants and auditors need to use vast amounts of data to not only report on the past but also provide timely assurance and insights about the business' future. There is a need to employ dynamic analytics or visualization tool to increase the impact of their opinions and recommendations. Thus, it is imperative that the accounting profession adopts and implements dynamic reporting and visualization techniques 
that deal with the big-data problem and produce results that enhance the ability to make an impact and gain influence.

\section{Conclusion}

The use of automation, big data and other technological advances such as machine learning will continue to grow in accounting and audit, producing important business intelligence tools that provide historical, current and predictive views of business operations in interactive data visualizations. Business intelligence systems allow accounting professionals to make better decisions by analyzing very large volumes of data from all lines of business, resulting in increased productivity and accuracy and better insights to make more informed decisions. The built-in, customizable dashboards allow for real-time reporting and analysis, where exceptions, trends and opportunities can be identified and transactional data drilled down for greater detail.

Analytics, artificial intelligence, and direct linkages to clients' transaction systems can allow audits to be a continuous rather than an annual process, and material misstatements and financial irregularities can be detected in real time as they occur, providing near real-time assurance. Audit team members could reduce the performance of repetitive low-level tasks in verifying transactional data and be involved in high-value tasks by focusing their efforts on the interpretation of the results produced by machines. With adequate understanding of the wider business and economic environment in which the client entity operates, from changes in technology or competition, auditors are more able to assess the reasonableness of the assumptions made by management, instead of just focusing on mechanical details. Such improvements will enhance the application of professional skepticism in framing auditor's judgments when performing risk assessment procedures and consequently, design an audit strategy and approach that will be responsive to the assessed risks of material misstatement.

As audits become substantially more automated in the future, auditors could also provide valuable insights to the clients, such as how the clients' performances fare in comparison with similar companies on key metrics and benchmarks, providing value-added services in addition to audit service. Eventually, it will be the investing public who will benefit from higher quality, more insightful audits powered by machine learning and big data analysis across clients and industries.

\section{Acknowledgements}

Mui Kim Chu is senior lecturer at Singapore Institute of Technology. Kevin Ow Yong is associate professor at Singapore Institute of Technology. We wish to thank Khin Yuya Thet for her research assistance. All errors are our own.

\section{Conflicts of Interest}

The authors declare no conflicts of interest regarding the publication of this paper. 


\section{References}

Alawadhi, A. (2015). The Application of Data Visualization in Auditing. Rutgers, The State University of New Jersey

Davenport, T. H. (2016). The Power of Advanced Audit Analytics Everywhere Analytics. Deloitte Development LLC.

https://www2.deloitte.com/content/dam/Deloitte/us/Documents/deloitte-analytics/usda-advanced-audit-analytics.pdf

Dickey, G., Blanke, S., \& Seaton, L. (2019). Machine Learning in Auditing: Current and Future Applications. The CPA Journal, 89, 16-21.

Eaton, T., \& Baader, M. (2018). Data Visualization Software: An Introduction to Tableau for CPAs. The CPA Journal, 88, 50-53.

Haq, I., Abatemarco, M., \& Hoops, J. (2020). The Development of Machine Learning and its Implications for Public Accounting. The CPA Journal, 90, 6-9.

IAASB (2018). Exploring the Growing Use of Technology in the Audit, with a Focus on Data Analytics. International Auditing and Assurance Standards Board.

Lim, J. M., Lam, T., \& Wang, Z. (2020). Using Data Analytics in a Financial Statement Audit. IS Chartered Accountant Journal.

Martens, D., Bruynseels, L., Baesens, B., Willekens, M., \& Vanthienen, J. (2008). Predicting Going Concern Opinion with Data Mining. Decision Support Systems, 45, 765-777. https://doi.org/10.1016/j.dss.2008.01.003

McQuilken, D. (2019). 5 Steps to Get Started with Audit Data Analytics. AICPA. https://blog.aicpa.org/2019/05/5-steps-to-get-started-with-audit-data-analytics.html\#st hash.NSlZVigi.dpbs

Paltrinieri, N., Comfort, L., \& Reniers, G. (2019). Learning about Risk: Machine Learning for Risk Assessment. Safety Science, 118, 475-486.

https://doi.org/10.1016/j.ssci.2019.06.001

Skapoullis, C. (2018). The Need for Data Visualisation. ICAEW.

https://www.icaew.com/technical/business-and-management/strategy-risk-and-innova tion/risk-management/internal-audit-resource-centre/the-need-for-data-visualisation

Tschakert, N., Kokina, J., Kozlowski, S., \& Vasarhelyi, M. (2016). The Next Frontier in Data Analytics. Journal of Accountancy, 222, 58.

Zabeti, S. (2019). How Audit Data Analytics Is Changing Audit. Accru. 